\title{
HIPPIDION OWEN 1869 (MAMMALIA, PERISSODACTYLA) EN SEDIMENTOS DEL PLEISTOCENO TARDIO DE LA LOCALIDAD BARRO NEGRO (JUJUY, ARGENTINA) ${ }^{(1)}$
}

\author{
M. T. Alberdi $\left(^{*}\right)$, J. Fernández (**), A. N. Menegaz (***) y J. L. Prado (***)
}

\section{RESUMEN}

El presente estudio comprende los hallazgos realizados en la localidad de Barro Negro (Humahuaca, Jujuy, Argentina). Incluye una descripción y comentarios de los rasgos sobresalientes del material, así como aspectos estratigráficos y cronológicos relacionados.

Palabras clave: Perissodactyla, Hippidion, Pleistoceno tardío, Argentina.

\section{ABSTRACT}

The present study includes the recent finds in the Barro Negro locality (Humahuaca, Jujuy, Argentina). Description and most important coments of this remains are developed. Chronological and stratigraphycal aspects are also included.

Key words: Perissodactyla, Hippidion, Later Pleistocene, Argentina.

\section{Introducción}

Desde hace más de un siglo la literatura paleontológica se ha ocupado de los équidos sudamericanos, tal como lo atestiguan, entre otros, los excelentes trabajos monográficos publicados (Burmeister, 1875, 1889; Sefve, 1912; Boule et Thevenin, 1920; Hoffstetter, 1952).

Esta variedad de estudios ha generado una gran cantidad de denominaciones taxonómicas, muchas de las cuales han sido fundadas con materiales aislados o incompletos.

Los hallazgos realizados recientemente por uno de los autores (Fernández, $1985 \mathrm{a}, 1985$ b), en la localidad de Barro Negro (Humahuaca, Jujuy, Argentina), abren una nueva perspectiva. En efecto, estos restos, numerosos y bien conservados, permiten hipotetizar acerca de las variaciones morfológicas que deben tenerse en cuenta a la hora de su determinación.

El presente estudio tiene por finalidad dar a cono- cer estos restos, así como ponderar las variaciones osteométricas observadas.

\section{Situación geográfica}

La localidad de Barro Negro, situada en el departamento de Humahuaca (provincia de Jujuy, República Argentina), se encuentra aproximadamente a los $23^{\circ} \mathrm{S}$ y $65^{\circ} 37^{\prime} \mathrm{W}$, a $10 \mathrm{~km}$ de distancia de la estación ferroviaria de Tres Cruces (fig. 1).

Regionalmente esta localidad corresponde a la Cordillera Oriental, límite E de la Puna. Esta región se caracteriza por presentar cadenas montañosas alineadas en dirección N-NE, separadas por valles profundos. La altitud media es de $\mathbf{4 . 0 0 0}$ metros.

El clima corresponde al característico de un desierto de altura: seco, con precipitaciones que oscilan entre los 300 y $750 \mathrm{~mm}$ anuales y con una marcada amplitud térmica diurna. Durante el verano es

(1) Este trabajo se ha realizado dentro del Convenio bilateral CONICET-CSIC, «Correlaciones del Cuaternario reciente entre Sudamérica y Europa con énfasis especial en las faunas españolas y argentinas".

(*) Museo Nacional de Ciencias Naturales. José Gutiérrez Abascal, 2. 28006 Madrid.

(**) Instituto Nacional de Antropología, 3 de Febrero 1370 (1426). Buenos Aires (Argentina).

(***) CIC Museo de la Plata. Paseo del Bosque, s/n. La Plata 1900 (Argentina). 


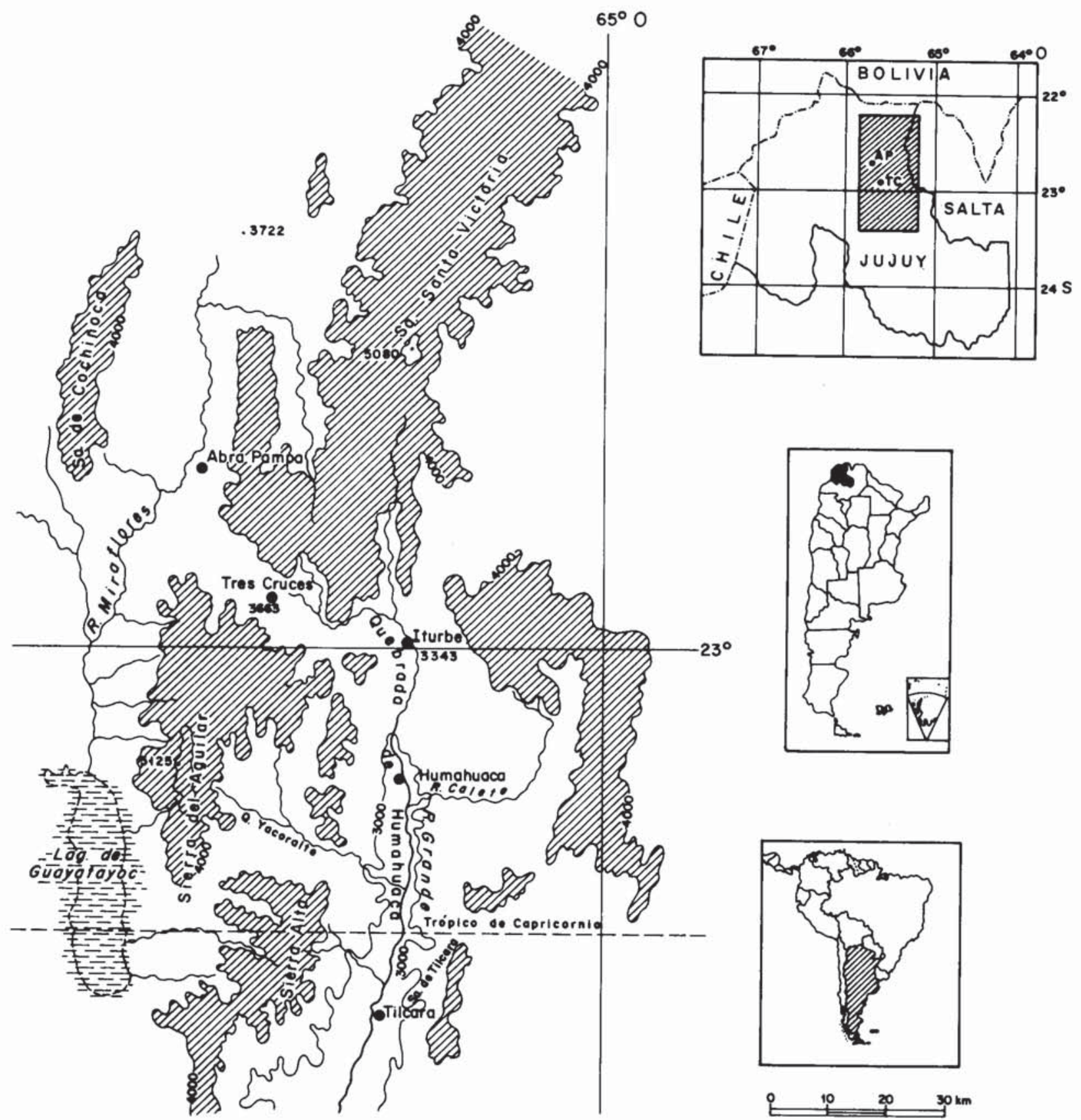

Fig. 1.-Situación geográfica de la región donde se efectuaron los hallazgos (Rep. Argentina).

templado a frío y muy frío durante el invierno (Turner y Méndez, 1979; Turner y Monn, 1979).

\section{Estratigrafía}

Los materiales estudiados provienen de los barrancos de la Quebrada de las Piedras elaborada por un tributario del arroyo Barro Negro.
La secuencia estratigráfica comienza con un depósito verdoso de granulometría limo-arenoso, sin base expuesta. Continúa con un sedimento negro constituido por materiales turbificados de una potencia de hasta $2,5 \mathrm{~m}$. Este nivel presenta a menudo intercalaciones de un sedimento limo-arenoso de color verde que alterna con capas turbosas marrones.

Lateralmente, y en forma discontinua a estos sedimentos, se observan depósitos de margas parcialmente 


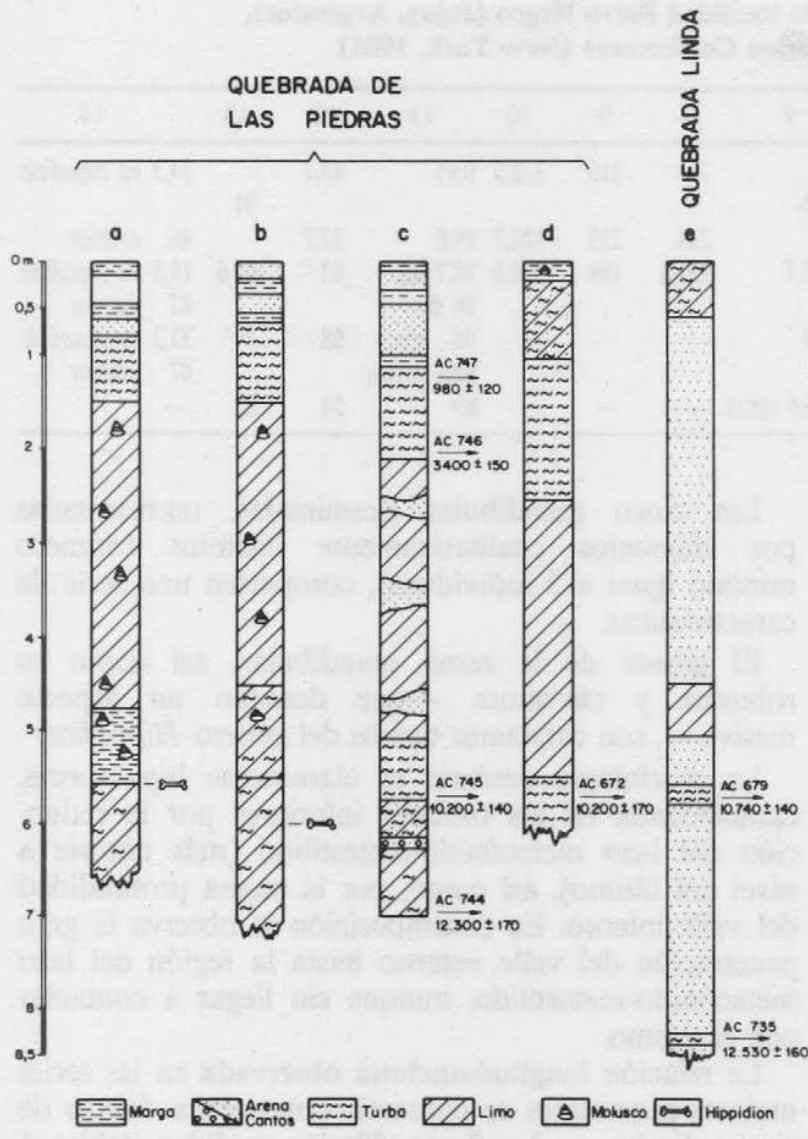

Fig. 2.-Diagrama comparativo de las columnas estratigráficas obtenidas en la localidad Barro Negro (Jujuy, Argentina).

diatomeaceas, con abundante Lymanea viatrix (Fernández, 1985 b).

Completando la secuencia se suceden varios niveles sedimentarios, los cuales han sido tratados previamente por uno de los autores (Fernández, 1985 a) (fig. 2).

Los restos fósiles de équidos que motivan el presente trabajo provienen tanto de los niveles turbosos como de sus variaciones laterales.

Dataciones radiométricas efectuadas sobre sedimentos turbosos de esta localidad arrojan las siguientes edades radiocarbónicas:

\begin{tabular}{|c|c|}
\hline Muestras & Edad \\
\hline AC. 672 & $10.200 \pm 170 \mathrm{BP}$ \\
\hline AC. 679 & $10.740 \pm 140 \mathrm{BP}$ \\
\hline AC. $735 \ldots \ldots \ldots$ & $12.530 \pm 160 \mathrm{BP}$ \\
\hline AC. $745 \quad \ldots \ldots \ldots$ & $10.200 \pm 140 \mathrm{BP}$ \\
\hline AC. $744 \ldots \ldots \ldots$ & $12.300 \pm 170 \mathrm{BP}$ \\
\hline
\end{tabular}

Como se observa, estos fechados guardan una estrecha relación entre sí.
La evaluación de estos fechados tiene un carácter preliminar, estando sujeta al desarrollo de futuras investigaciones en la zona.

Mayores detalles acerca de la cronología, características de las muestras analizadas y detalles de las técnicas utilizadas se encuentran en Fernández, 1985 b.

\section{Materiales y métodos}

El material procedente de la localidad de Barro Negro, objeto de este estudio, se encuentra depositado en las colecciones de paleontología de vertebrados del Museo de La Plata (MLP), y consiste en:

MLP 85-VII-1-1. Mandíbula completa con series dentarias completas (lám. I, fig. e; fig. 3).

MLP 85-VII-1-2. Mandíbula incompleta, serie molariforme izquierda completa; $\mathrm{P}_{2}-\mathrm{P}_{4}$ derechos, sínfisis (lám. I, fig. a).

MLP 85-VII-1-3. Rama mandibular izquierda y sínfisis; serie molariforme izquierda deteriorada.

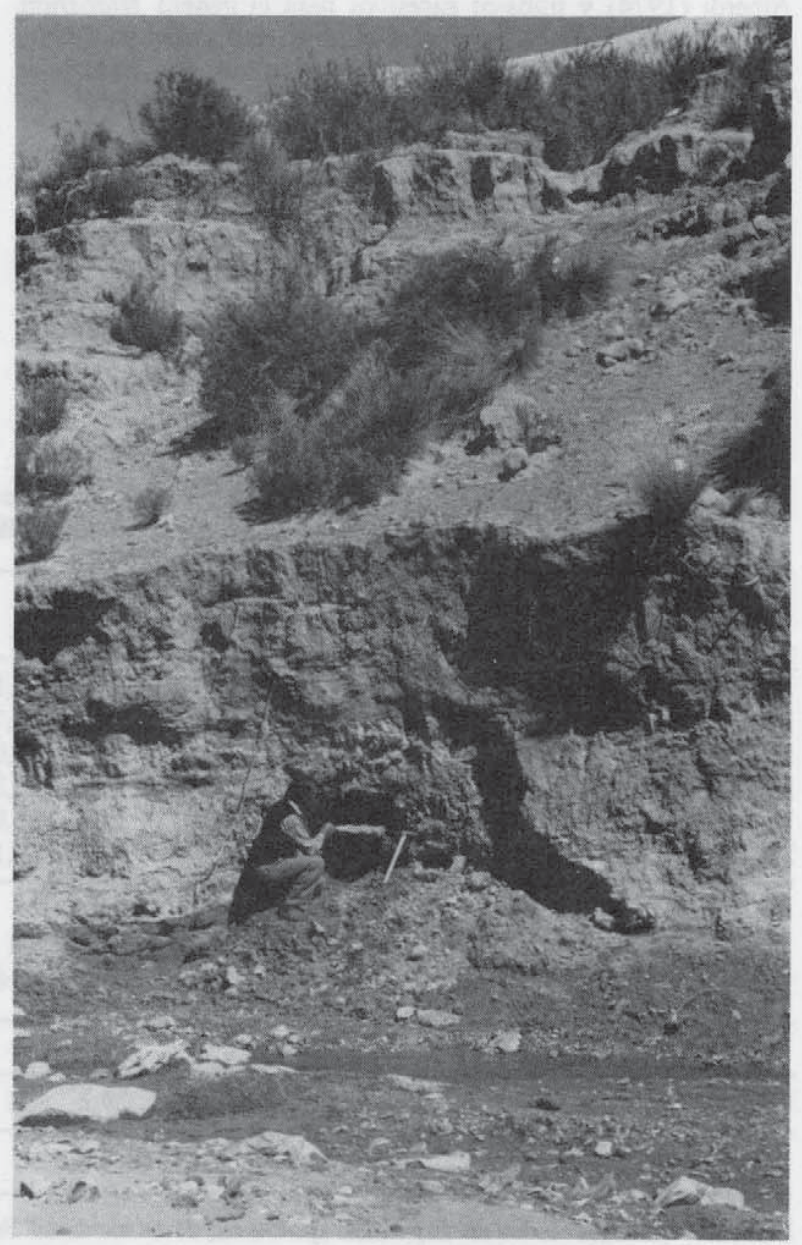

Fig. 3.-Barranca W de la Quebrada de las Piedras. Lugar donde se extrajo el ejemplar número MLP 85-VII-1-1 (Jujuy, Argentina). 
Tabla 1.-Dimensiones de las mandíbulas, de la localidad Barro Negro (Jujuy, Argentina), siguiendo la nomenclatura de la «Hipparion Conference" (New York, 1981)

\begin{tabular}{|c|c|c|c|c|c|c|c|c|c|c|c|c|c|c|}
\hline & 1 & 2 & 3 & 4 & 5 & 6 & 7 & 8 & 9 & 10 & 11 & 12 & 13 & 14 \\
\hline MLP 85-VII-1-1 dr. ... & 421 & $77,7 \mathrm{I}_{3}$ & 85,8 & 83 & 168,8 & 127 & 76 & 219 & 210 & 121,5 & 93,5 & 65,7 & 91 & 34,3 en su \\
\hline MLP 85-VII-1-1 izq. ... & 421 & $72,0 \mathrm{I}_{3}$ & 85 & 82 & 169,9 & 129 & & 226 & 215 & 123,7 & 89,0 & 57,7 & & 46 exterior \\
\hline ALP 85-VII- & 405 & $71 \mathrm{I}_{3}$ & 81 & 78,5 & 161 & 127 & 66,7 & 195,5 & 189 & 110,5 & $\begin{array}{l}98,7 \mathrm{izq} . \\
96 \mathrm{dr} .\end{array}$ & 63 & 80,6 & $\begin{array}{l}19,8 \text { en superficie } \\
47 \text { exterior }\end{array}$ \\
\hline MLP 85-VII-1-3 izq. ... & - & $62,5 \mathrm{I}_{3}$ & 82,5 & 81 & 162 & - & 59 & - & - & - & $\begin{array}{l}95 \text { aprox. } \\
92,5 \text { dentro }\end{array}$ & 58 & 74 & $\begin{array}{l}33,2 \text { en superficie } \\
47 \text { exterior }\end{array}$ \\
\hline MLP 85-VII-1-4 dr. ... & - & $65 \mathrm{I}_{3}$ & - & - & - & - & 56,6 aprox. & & - & - & 89 & 54 & 82 & - \\
\hline
\end{tabular}

MLP 85-VII-1-4. Fragmento de rama mandibular derecha y sínfisis; $\mathbf{P}_{2}-\mathbf{P}_{4}$ derechos

MLP 85-VII-1-5. Sínfisis (muy alterada).

MLP 85-VII-1-6. Metatarsiano III derecho (lám. I, figs. c y d).

MLP 85-VII-1-8. Tibia derecha incompleta, sin articulación proximal (lám. I, fig. 8).

En cuanto al método a seguir, se utilizó el elaborado por Alberdi (1974) y trabajos sucesivos para el género Hipparion, así como las recomendaciones y normas elaboradas en cuanto a la toma de parámetros y caracteres métricos, con motivo de "Hipparion Conference" celebrada en New York, 1981 (en prensa), basado en parte en Eisenmann (1979).

\section{Paleontología sistemática}

Orden: Perissodactyla (Owen, 1848).

Subfamilia: Equinae (Steinmann and Döderlein, 1890).

Género: Hippidion (Owen, 1869).

Hippidion sp.
Las cinco mandíbulas examinadas, representadas por elementos cualitativamente distintos (número mínimo igual a 5 individuos), comparten una serie de características.

El grosor de la rama mandibular, así como su robustez y curvatura -que denotan un aspecto masivo-, son caracteres típicos del género Hippidion.

La morfología dentaria es claramente hipidiforme, caracterizada en los molares inferiores por la reducción del lazo metacónido-metastilido (más notoria a nivel del último), así como, por la escasa profundidad del valle interno. En contraposición se observa la gran penetración del valle externo hasta la región del lazo metacónido-metastílido, aunque sin llegar a contactar con él mismo.

La relación longitud-anchura observada en las series molares-premolares se conserva homogénea dentro de ciertos límites en las 3 mandíbulas medidas (tablas 1 y 2). Por el contrario, la forma y longitud de la sínfi-

Tabla 2.-Dimensiones en mm de los dientes correspondientes a las mandibulas de Hippidion sp. Owen 1869, de la localidad Barro Negro (Jujuy, Argentina)

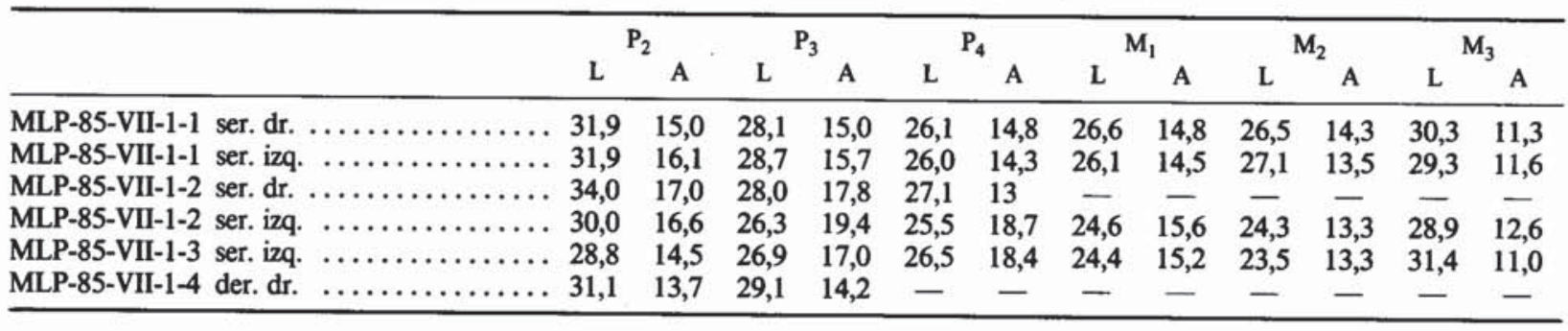

Tabla 3.-Dimensiones de Mt III der. de Hippidion sp. Owen 1869, de Barro Negro (Jujuy, Argentina)

\begin{tabular}{ccccccccccccccc}
\hline & 1 & 2 & 3 & 4 & 5 & 6 & 7 & 8 & 9 & 10 & 11 & 12 & 13 & 14 \\
\hline MLP 85-VII-1-6-Mt III dr. $\ldots$ & 190,5 & 184 & 32,1 & 29,2 & 51 & 35 & - & 10 & - & 45,6 & 42,1 & 30,8 & 22 & 26,5 \\
\hline
\end{tabular}

Lámina I.-Restos fósiles de Hippidion sp. Owen, de la localidad Barro Negro (Jujuy, Argentina). Todos a la misma escala: Fig. a: Vista oclusal de la mandibula (MLP-85-VII-1-2). - Fig. b: Vista anterior de la tibia derecha (MLP 85-VII-1-8). - Fig. c: Vista anterior del Mt III derecho (MLP 85-VII-1-6).-Fig. d: Vista posterior del Mt III derecho (MLP 85-VII-1-6).-Fig. e: Vista oclusal de la mandibula (MLP-85-VII-1-1) 


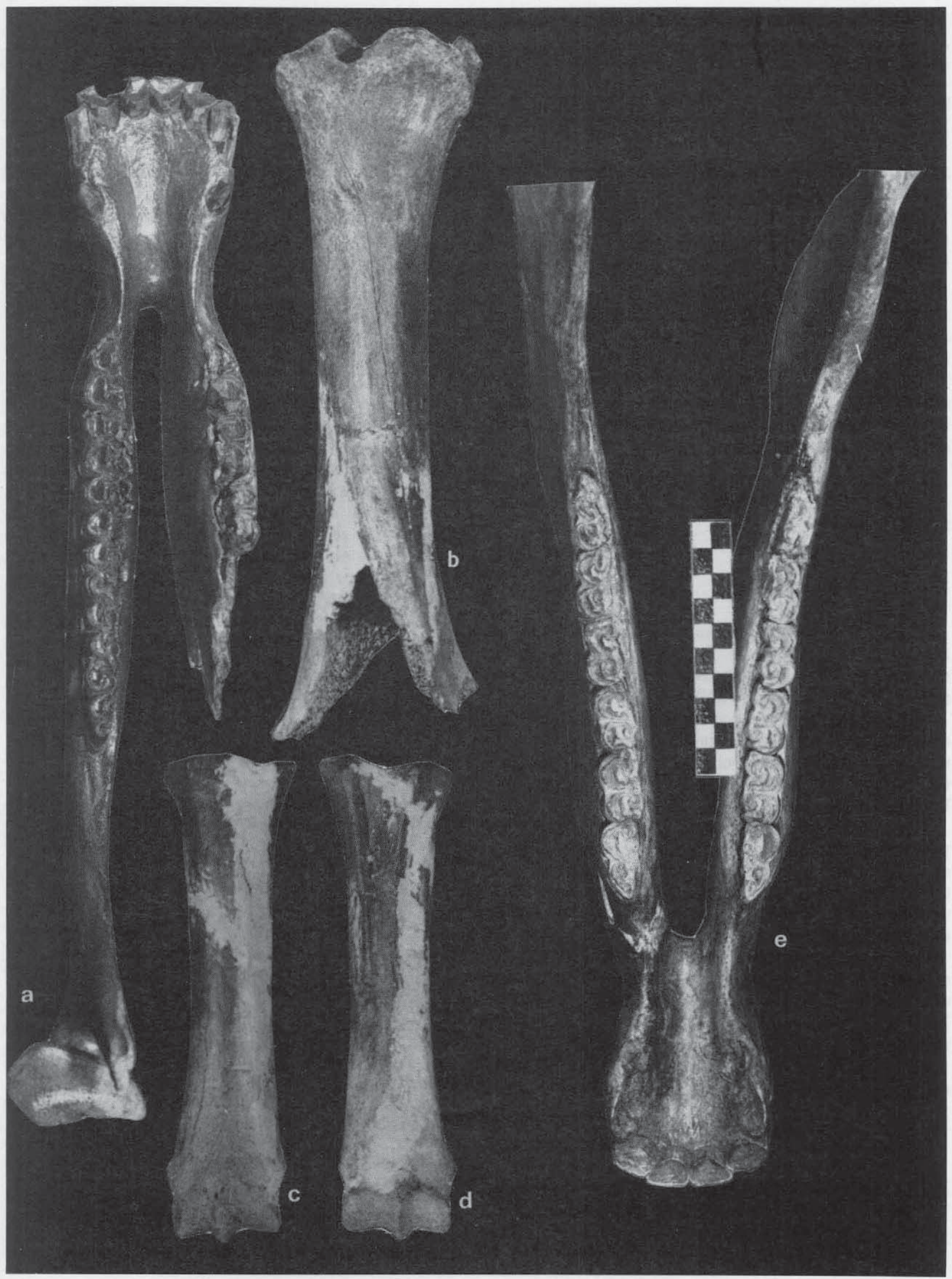


Tabla 4.-Dimensiones de la Tíbia de Hippidion sp. Owen 1869. Barro Negro (Jujuy, Argentina)

\begin{tabular}{|c|c|c|c|c|c|c|c|c|c|c|}
\hline & & 1 & 2 & 3 & 4 & 5 & 6 & 7 & 8 & 9 \\
\hline MLP $85-$ VII-1-8 dr. & $\ldots \ldots \ldots \ldots$ & - & - & 41,7 & 32 & - & - & 76,3 & 46,5 & - \\
\hline
\end{tabular}

sis mandibular presenta una gran variabilidad en los cinco individuos considerados.

Respecto de los elementos del esqueleto postcraneal, escasamente representados, se puede señalar lo siguiente:

El Mt III derecho corresponde a un individuo relativamente joven, encontrándose deformado en su extremidad proximal. En comparación con el carácter masivo de la mayoría de los metápodos del género Hippidion, nuestro ejemplar manifiesta una cierta gracilidad (ver tabla 3). Conserva el Mt IV soldado hasta la mitad de su longitud, característica ésta del género Hippidion (tabla 3).

Con respecto a la tibia, el ejemplar presenta igualmente una mayor gracilidad que la característica del grupo (tabla 4).

\section{Discusión y conclusiones}

A partir de los rasgos más sobresalientes que se acaban de mencionar, es factible hipotetizar que el conjunto de los materiales considerados pertenecen al género Hippidion Owen, 1869.

Sin embargo, basándonos en la historia taxonómica de este grupo, no es aconsejable realizar una determinación por debajo del nivel genérico hasta tanto no se cuente con una clara descripción de las especies involucradas en el mismo.

Los especímenes considerados en este trabajo nos ponen sobre aviso acerca de las variaciones intraespecíficas que se pueden observar en función de la talla y la edad. Consecuentemente, previo a una determinación específica, es necesario tener en cuenta ésta y otras particularidades.

Esta muestra permite establecer, en función del desgaste dentario, dos rangos de edades. El de menor edad, representado por los ejemplares MLP 85-VII-1-1, MLP 85-VII-1-2 y MLP 85-VII-1-4 y el de mayor, representado por el ejemplar MLP 85-VII-1-3.

Las variaciones métricas de los caracteres observados en estas 2 entidades son las siguientes:

1. La longitud de la sínfisis (13) decrece desde el rango inferior al superior. Posiblemente este sea el rasgo más notorio.

2. La serie dentaria (5) sufre un acortamiento a medida que se incrementa la edad; tanto en la serie premolar (3) como en la molar (4). Esta reducción se manifiesta más nítidamente en la longitud y anchura del $\mathrm{P}_{2}$, y en la longitud de el $\mathrm{P}_{3}$ y $\mathrm{M}_{2}$

3. El diastema (14) sufre el mismo acortamiento progresivo, inversamente proporcional a la edad del individuo.

4. El cuerpo mandibular más robusto corresponde al ejemplar más joven (MLP 85-VII-1-1) si bien todas las mandíbulas son robustas.

5. La altura de la mandíbula se mantiene constante en ambas clases.

Frecuentemente, el hallazgo de restos fósiles aislados y fragmentarios dificultan su análisis detallado.

En el caso de la sistemática de los hipidiformes, los restos provenientes de distintas localidades ponen de manifiesto una gran homogeneidad estructural (Prado, 1984; Alberdi et al., (1986). Sin embargo, esta homogeneidad no se ve reflejada en la nomenclatura utilizada en la literatura específica (Burmeister, 1875, 1889; Moreno, 1891; Sefve, 1912; Boule et Thevenin, 1920; Hoffstetter, 1952; Fernández de Alvarez, 1957, 1959; Macfadden and Skinner, 1979). Por ello nos limitamos, por el momento, a asignar estos materiales a nivel genérico: Hippidion sp., en espera de un estudio exhaustivo que involucre la totalidad de los équidos sudamericanos.

\section{AGRADECIMIENTOS}

La colaboración de J. Arroyo por los dibujos, Teresa Montero por el mecanografiado, Mario Cozuol por la elaboración de la fotografia, así como al Laboratorio de Fotografia del Museo Nacional de Ciencias Naturales (Madrid).

\section{Referencias}

Alberdi, M. T. (1974): El género Hipparion en España. Nuevas formas de Castilla y Andalucía, revisión e historia evolutiva. Trabajos sobre Neógeno-Cuaternario, 1, 1-164.

Alberdi, M. T.; Menegaz, A. N., y Prado, J. L. (1986): Formas terminales de Hippidion (Mammalia, Perissodactyla) de los yacimientos del Pleistoceno tardio-Holóceno de la Patagonia (Argentina y Chile). Estudios geol., 42 (en este volumen).

Boule, M. y Thevenin, A. (1920): Mammifères fossiles de Tarija. París Imprimerie Nationale, 1-255.

Burmeister, G. (1875): Los caballos fósiles de La Pampa Argentina. Imp. "La Tribuna». Buenos Aires, Argentina (Bilingüe), 1-88.

Burmeister, G. (1889): Los caballos fósiles de La Pampa. Argentina Suplemento. Imprenta "La Universidad». Buenos Aires. Argentina (Bilingüe), 1-65.

Eisenmann, V. (1979): Les Chevaux (Equus sensu lato) fossiles 
et actuels. Etude Craniologique et odontologique. Thèse de Doct. d'Etat. Univ. Pierre et Marie Curie. París VI, 1-444.

Fernández, J. (1985 a): Nuevas evidencias cronológicas y paleoambientales del Holóceno (intervalo 10.200-510 a A.P.) de la Puna jujeña: los derrames petrolíferos de Barro Negro y su contenido arqueológico y paleofaunístico (insectos, pájaros y mamíferos). MS.

Fernández, J. (1985 b): Reemplazo del caballo americano (Perissodactyla) por camélidos (Artiodactyla) en estratos del límite pleistocénico-holocénico de Barro Negro, Puna de Jujuy, Argentina. Implicancias paleoambientales, faunísticas y arqueológicas. Relaciones de la Soc. Argentina de Antrop., N. A. 1984-1985. Bs. As., 16, 137-152.

Fernández de Alvárez, E. (1957): Hipohippidium humahuaquense nov. sp. Ameghiniana 1, 85-95.

Fernández de Alvarez, E. (1959): Algunas observaciones sobre Parahipparion y descripción de una nueva especie. Rev. Asociación Geológica Argentina, 14, 239-250.

Hoffstetter, R. (1952): Les mammifères Pleistocènes de la République de L'Equateur. Mém. Soc. Geol. France, 31, 1-391.
Macfadden, B. J. y Skinner, M. F. (1979): Diversification and Biogeography of the One-Toed Horses Onohippidium and Hippidion. Postilla, 175, 1-10.

Moreno, F. P. (1891): Onohippidium muñizi. Breve noticia sobre los restos fósiles de un género nuevo de la familia de los Equidae conservados en el Museo de La Plata. Rev. Mus. La Plata, 2, 65-71.

Prado, J. L. (1984): Fenética de los metatarsianos de taxa fósiles Hippidion, Owen; Onohippidium, Moreno; Parahipparion, C. Ameghino, y Equus (Amerhippus), Linne (Mammalia. Perissodactyla). C.I.P.F.E.-C.E.D. Orione Cont. Biol., Montevideo 11, 11-15.

Sefve, I. (1912): Die fossilen Pferde Südamerikas. Sve. Vet. Akad. Handl., 48, 1-185.

Turner, I. C. M. y Méndez, V. (1979): Puna in Geologia Regional Argentina. I. Acad. Nac. Ciencias Córdoba, 13-56.

Turner, J. C. M. y Mon, R. (1979): Cordillera Oriental, en Geología Regional Argentina II. Acad. Nac. Ciencias, Córdoba, 57-94.

Recibido el 29 de septiembre de 1986 Aceptado el 10 de diciembre de 1986 